\title{
Marginal adaptation of composite overlays based on two methods of impressions; Conventional technique and digital scanner. An in vitro Study
}

\author{
Vicente José Faus Llácer ${ }^{1 *}$, Carla García Cuesta ${ }^{1}$, René Botello Torres ${ }^{1}$, Ignacio Faus Matoses ${ }^{2}$, Teresa Alegre Domingo ${ }^{1}$ and Vicente Faus \\ Matoses ${ }^{1}$ \\ ${ }^{1}$ Master of Restorative Dentistry and Endodontics, Department of Stomatology, Medicine and Dental School, Gascó Oliag 1, 46010 Valencia, Spain \\ ${ }^{2}$ Master of Orthodontics, Department of Stomatology, Medicine and Dental School, Gascó Oliag 1, 46010 Valencia, Spain
}

\begin{abstract}
Objective: Evaluation of marginal adaptation of composite overlays with different impression techniques; one with conventional silicone (Express ${ }^{\mathrm{TM}}$ VPS Impression Material, 3M ESPE, Seefeld, Germany) compared to a digital impression with the scanner True Definition (3M ESPE, Seefeld, Germany).

Material and method: 80 extracted third molars divided into two groups $(n=40)$. MOD cavities were prepared in the teeth to obtain composite overlays. One group had impressions made with silicone and the restorations made by the laboratory technician; in the other group an intraoral scanner took the impressions and the restorations were made by CAD/CAM. Under 32x of magnification, images of the vestibular, lingual, mesial and distal surfaces were captured and sent to a computer with a screen ruler. Firstly, the passive adjustment was evaluated. The gaps were measured in micrometres along a perpendicular line from the internal surface of the restoration to the external surface of the tooth. With Power Point computer application (Microsoft office, 2010) the distance between tooth/restoration was measured in micrometres. After cementation, the marginal adaptation was evaluated. ANOVA analysis and Bonferroni test were used to achieve the mean of the marginal adaptation.
\end{abstract}

Results: The scanner group achieved better results. The most significant difference was achieved just after cementation ( $\mathrm{p}=0.022)$. The best results were obtained in the occlusal surface ( $\mathrm{p}=0.016$ scanner; $\mathrm{p}<0.001$ silicone); gingival angle achieved less adaptation in both groups (scanner $184.69 \mu \mathrm{m} ;$ silicone $242.29 \mu \mathrm{m}$ ).

Conclusions: Digital scanners are an alternative with favourable results in impression taking.

\section{Introduction}

Indirect composite restorations such as overlays are used to cover endodontically treated teeth and thus protect the teeth against fracture [1]. They copy the occlusal anatomy and the marginal adaptation of the tooth [2], preventing the entrance of fluids and bacteria inside the restoration, reducing sensitivity, plaque accumulation [3], bacterial invasion and secondary caries $[4]$.

A link has been demonstrated between the material, the impression technique and the marginal adaptation [5]. Impression materials have a propensity to suffer three-dimensional changes because of the chemical reactions and this could adversely affect the marginal adaptation and microleakage [6]. There are three ways of taking dental impressions; conventional impression with silicone or polyether, conventional impression with the posterior scans of the impression and intraoral digital impression [6].

Improved scanner technology over the last 20 years means that there is now an alternative for taking intraoral impressions [7]. Their use has resulted in an improvement in impression taking, providing a better marginal adaptation compared to the conventional technique and leading to an improvement in patient satisfaction [7-9].

A variety of scanners are available; some of them require preparation of the tooth prior to taking the impression. The Lava C.O.S (Lava Chairside Oral Scanner; 3M ESPE, Seefeld, Germany) requires the tooth surface to be covered with titanium dioxide in order to avoid absorption and light refraction [10]. On the other hand, the scanner CEREC AC (Sirona Dental Systems GmbH, Benshein, Germany) needs to work on a matt surface, achieved by applying a layer of Optispray over the tooth [11]. However, there are other scanners such as i-Tero (Cadent Inc, Carlstadt, USA) where scans can be taken without any prior preparation of the teeth [6].

The scanner True Definition (3M ESPE, Seefeld, Germany) has been in the market for only a short time. This system needs the tooth to be prepared with titanium dioxide. It has a small, fixed camera with a huge accuracy in impression. The scanner True Definition (3M ESPE, Seefeld, Germany) has a different capturing system to other scanners like CEREC AC (Sirona Dental Systems GmbH, Benshein, Germany) or i-Tero (Cadent Inc, Carlstadt, USA) which capture individual 3D images in a short time lapse and which are then combined to give a complete 3D model $[6,10]$. The Lava C.O.S (Lava Chairside Oral

Correspondence to: Vicente José Faus Llácer, Master of Restorative Dentistry and Endodontics, Department of Stomatology, Medicine and Dental School, Gascó Oliag 1, 46010 Valencia, Spain, Tel: +34 690307237; E-mail address: fausvj@uv.es

Key words: composite overlays, marginal gap, scanners, silicone

Received: August 10, 2016; Accepted: September 09, 2016; Published: September 12, 2016 
Scanner; 3M ESPE, Seefeld, Germany) system works in a similar way to the True Definition scanner (3M ESPE, Seefeld, Germany). These scanners consist of $3 \mathrm{D}$ video that capture 20 images per second in real time $[6,10]$. Because of this the scanner True Definition (3M ESPE, Seefeld, Germany) is recommended for taking impressions of overlays, crowns, bridges, implant prosthesis and orthodontics. These intraoral scanners complement CAD/CAM techniques for digital design and manufacture, increasing the speed of design and creation [9]. With this method, the scanner information is rapidly transmitted to a milling machine in the laboratory or in the same clinic [11].

The aim of this paper was to analyse the marginal gap in composite overlays; one obtained by a conventional impression with silicone (Express $^{\mathrm{nm}}$ VPS Impression Material, 3M ESPE, Seefeld, Germany) compared to other with digital impression using the scanner True Definition (3M ESPE, Seefeld, Germany).

\section{Material and method}

A research project was carried out as part of a Master in Endodontic and Restorative Dentistry of the Stomatology Department, University of Medicine and Dentistry, University of Valencia.

80 extracted third molars were selected which were free from caries. The teeth were embedded in plaster $2 \mathrm{~mm}$ above the cementoenamel junction, in groups of 10, imitating a dental arch. To prepare the overlays a diamond bur was used (ISO 806314544514016, Komet Dental, Lemgo, Germany), making divergent cavities of $2 \mathrm{~mm}$ in the occlusal surface $[4,5,12]$. The marginal chamfers were $\geq 1 \mathrm{~mm}$. The axial walls were prepared and the transition between gingival and proximal were rounded and smoothed with the same bur.

The teeth were divided into 2 working groups according to the impression method used $(n=40)$.

\section{- Group 1 (conventional silicone)}

A conventional impression with silicone (Express ${ }^{\text {tix }}$ VPS Impression Material, 3M ESPE, Seefeld, Germany) was taken of each group of 10 teeth, using the wash technique, mix heavy and light silicone in metallic trays. The impressions were sent to the laboratory where the plaster was removed, the teeth were separated and overlays were made by a laboratory technician using indirect composite (Gradia indirect, GC, Tokyo, Japan).

\section{- Group 2 (intraoral scanner)}

Impressions of the groups of 10 were taken with the intraoral scanner (True Definition Scanner, 3M ESPE, Seefeld, Germany). The surface of each tooth was dried and covered with a thin layer of titanium dioxide. The digital impressions were sent directly to the laboratory technician from the scanner True Definition (3M ESPE, Seefeld, Germany), where they were relayed to the milling system Sirona MCXL that used composite blocks of Lava Ultimate CAD/CAM Restorative (3M ESPE, Seefeld, Germany) to produce the overlays.

\section{Marginal gap analysis}

All the overlays were observed using the binocular stereoscope MZ APO (Leica Microsystems Inc, Buffalo Grove, IL) at 32x of magnification; images of the vestibular, lingual, mesial and distal surfaces were captured (Figure 1 and 2). The images with a screen ruler incorporated were directly transmitted to a computer where they could be edited, measured and saved. First of all, the passive adjustment, considered to be the "adaptation" or closeness of fit of the overlay to

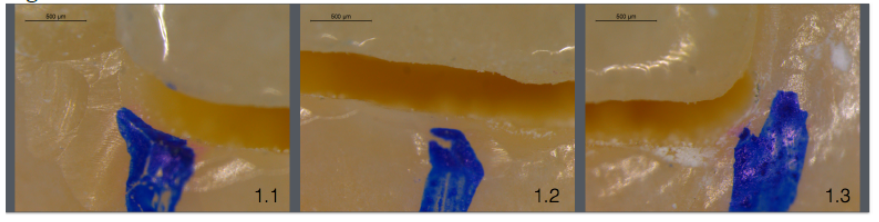

Figure 1. Images of the mesial surface in the silicone group before cementation under binocular stereoscopy.

1.1. Detail of the gingival angle, 1.2. Detail of the gingival floor, 1.3. Detail of the gingival angle.

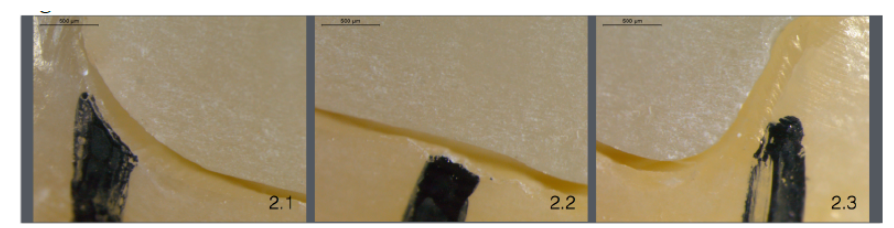

Figure 2. Images of the distal surface in the scanner group before cementation under binocular stereoscopy.

2.1. Detail of the gingival angle, 2.2. Detail of the gingival floor, 2.3. Detail of the gingival angle.

the model, was evaluated. The gaps were measured in micras $(\mu \mathrm{m})$ along a perpendicular distance from the inside surface of the overlay to the outside surface of the tooth. In addition, Power Point computer application (Microsoft office, 2010) was used to measure the distance between tooth/restoration; 12 points were measured; 6 in the occlusal surfaces (vestibular and palatal); 4 in the gingival angle and 2 in the gingival floor.

Next, all the restorations were sanded with titanium oxide. Ortofosforic acid 37\% Scotchbond Universal Etchant (3M ESPE, Seefeld, Germany) was applied for 15 seconds on the tooth and the overlay. They were then washed and dried. Next, a layer of silane (Relyx ceraminc primer, 3M ESPE, Seefeld, Germany) was applied to the inside surface of the restoration for 1 minute. Then, light curing adhesive Scotchbond Universal Adhesive (3M ESPE, Seefeld, Germany) was placed on the tooth and on the inside surface of the restoration. Finally, cementation was carried out with Relyx Luting Plus Cement (3M ESPE, Seefeld, Germany). The excess material was removed and the restorations were light cured for 40 seconds each side with a light Elipar $^{\text {ra }}$ S10 LED Curing Light, 3M ESPE, Seefeld, Germany). All the overlays were polished to remove any excess material. After cementation the marginal adaptation was evaluated using the criteria mentioned previously. All the information obtained was analysed statistically with the software SPSS 22 (Chicago, IL). The level of significance was set at $\mathrm{p}$-value $<0.05$. The ANOVA system was used to estimate the results for the variable "adjustment" and the Bonferroni test was used to compare the specific differences.

\section{Results}

The interface surface between tooth/restoration of both groups could be seen in Figure 3 and 4. The mean of the marginal gap values in the occlusal surface, gingival angle and gingival floor are shown in Table 1, before and after cementation. Before cementation there were no significant differences between scanner $(169.76 \mathrm{~m})$ and silicone $(190.89 \mu \mathrm{m}) \quad(\mathrm{p}=0.144)$; after cementation significant differences appeared between the scanner $(145.16 \mu \mathrm{m})$ and silicone group $(187.47$ $\mu \mathrm{m})(\mathrm{p}=0.022)$, with lower adaptation in the silicone group.

In relation to the three areas where measurements were taken around the tooth, the results were similar before cementation in group 1 and 2; the best result was obtained in the occlusal surface $(\mathrm{p}=0.070)$. 


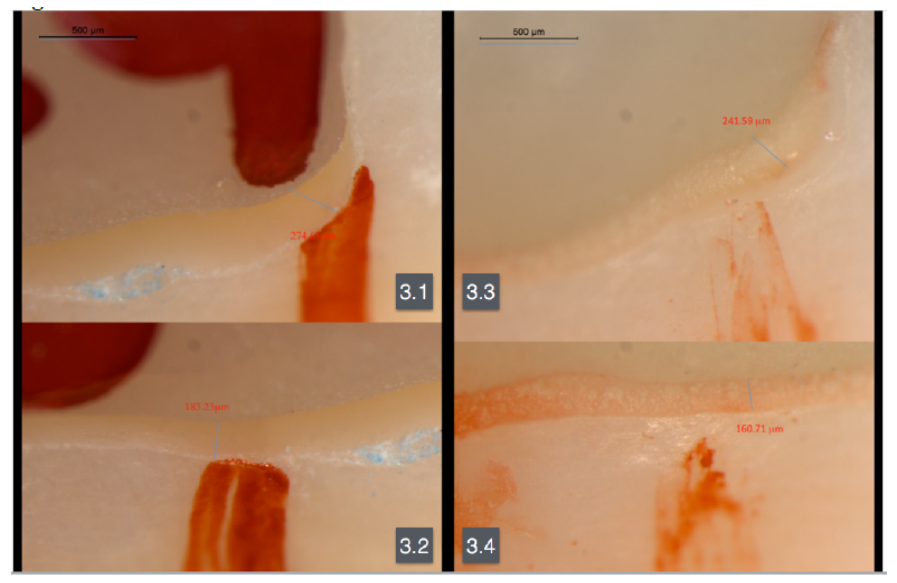

Figure 3. Silicone group.

3.1. Marginal gap analysis before cementation in the gingival angle, 3.2. Marginal gap analysis before cementation in the gingival floor, 3.3. Marginal gap analysis after cementation in the gingival angle, 3.4. Marginal gap analysis after cementation in the gingival floor.

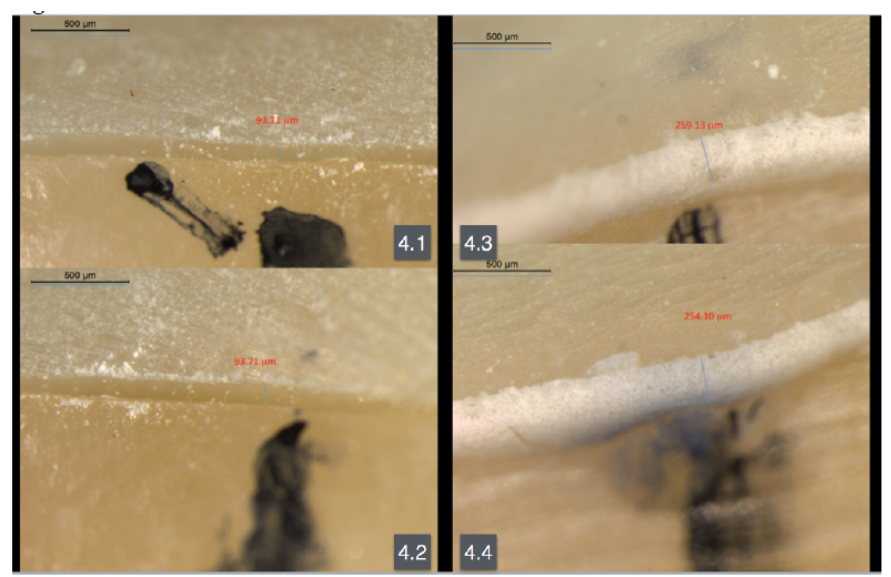

Figure 4. Scanner group

4.1. Marginal gap analysis before cementation in the occlusal surface, 4.2. Marginal gap analysis before cementation in the occlusal surface, 4.3. Marginal gap analysis after cementation in the occlusal surface, 4.4. Marginal gap analysis after cementation in the occlusal surface.

After cementation, the differences were more noticeable, especially in the gingival floor $(\mathrm{p}=0.022)$ and even more so in the gingival angle $(p=0.004)$; in the scanner group the results were higher.

\section{Discussion}

As new technologies are constantly evolving, it is crucial that modern devices such as intraoral digital scanners are compared to conventional techniques. This study was carried out to test the marginal adaptation of composite overlays; in the first group the impressions were taken conventionally with silicone (Express ${ }^{\mathrm{Tm}}$ VPS Impression Material, 3M ESPE, Seefeld, Germany) and then made by the laboratory technician; the other group used a digital system scanner True Definition (3M ESPE, Seefeld, Germany) to take the impressions and the $\mathrm{CAD} / \mathrm{CAM}$ process to made the restorations.

Depending on its smoothness, the cement used can affect the results of the adaptation [12], so in this study only one type of cement was used. Before cementation all samples had a gap with the purpose of holding the cement and to facilitate the polymerization shrinkage. Adhesive systems can reduce sensitivity, filtration and bacterial invasion, which could have an influence on the marginal adaptation [2].

There are few studies about the marginal adaptation of the composite overlays obtained by a digital system compared to conventional methods. This study showed that a statistically significant improvement was achieved after cementation in the group of the scanner versus the silicone group; the group of the scanner achieving a better adaption of $145.16 \mu \mathrm{m}$ compared to $187.57 \mu \mathrm{m}$ in the group with silicone; considering as marginal gap the distance between the external surfaces of the tooth to the internal surface of the restoration [5]. In this study, in both groups, the lower marginal adaptation was worse in the gingival angle compared to the occlusal point, which had achieved better adaptation. Similar results were obtained in a study of Zarrati et al. [5], where the gingival zones showed less favourable results with regard to adaptation.

$\mathrm{Ng}$ et al. [14] studied the adaptation of 30 crowns; in one group the impression had been taken with silicone and in the other with an intraoral scanner. The measurements in the group of the intraoral scanner $(48 \pm 25 \mu \mathrm{m})$ achieved better adaptation than the ones taken by silicone $(74 \pm 47 \mu \mathrm{m})$. Syrek et al. [3] stated that the intraoral impressions for crowns taken by Lava C.O.S system achieved an adaptation of $49 \mu \mathrm{m}$ in comparison to those impressions taken by silicone, which achieved a value of $71 \mu \mathrm{m}$. In the Seelbach et al. [11] study the results were $48 \pm 25 \mu \mathrm{m}$ for scanner and $60 \pm 30 \mu \mathrm{m}$ for silicone. Zarrati et al. [5] did not find any significant differences between the marginal gap before and after cementation $(\mathrm{p}=0.075 ; \mathrm{p}=0.766)$; nevertheless, in this study, after cementation the average adjustment of the scanner was significantly greater than the silicone $(\mathrm{p}=0.022)$. Additionally, several authors concluded that the digital method could be an alternative to the conventional one $[3,11,14]$.

Syrek et al. [3] affirmed that digital impression obtained significantly better marginal adaptation than conventional impressions. This could be because in the silicone group a model is made, whereas the restorations made by an intraoral scanner do not require such a model as the data goes straight to the milling machine. Consequently, each step in the process has an impact on the accuracy of the final product.

On the other hand, during the taking of digital impressions some difficulties arose in reproducing the interproximal surface accurately enough, although it was still easier to repeat scans for surfaces with poor precision than to remake an impression in the conventional way [8]. However, in this study, no difficulties were experienced in the taking of impressions of interproximal surfaces. Very little has been published about composite overlays made by way of a scanner. In this in vitro study, the imaging and the impressions where made under

Table 1. Mean marginal gap in scanner and silicone before and after cementation.

\begin{tabular}{|c|c|c|c|c|c|}
\hline & & \multicolumn{4}{|c|}{ GROUP } \\
\hline & & \multirow{2}{*}{\multicolumn{2}{|c|}{$\begin{array}{c}\text { Scanner }(\mu \mathrm{m}) \\
\text { CEMENT }\end{array}$}} & \multirow{2}{*}{\multicolumn{2}{|c|}{$\begin{array}{c}\text { Silicone }(\mu \mathrm{m}) \\
\text { CEMENT }\end{array}$}} \\
\hline & & & & & \\
\hline & & No & Yes & No & Yes \\
\hline \multirow{2}{*}{ OCLUSAL } & Mean & 120.44 & 111.63 & 99.15 & 106.70 \\
\hline & SD & 42.58 & 48.42 & 48.09 & 56.87 \\
\hline \multirow{2}{*}{$\begin{array}{l}\text { GINGIVAL } \\
\text { ANGLE }\end{array}$} & Mean & 227.12 & 184.69 & 254.65 & 242.29 \\
\hline & SD & 73.66 & 79.34 & 79.75 & 111.29 \\
\hline \multirow{2}{*}{$\begin{array}{l}\text { GINGIVAL } \\
\text { FLOOR }\end{array}$} & Mean & 161.71 & 139.16 & 218.88 & 213.42 \\
\hline & SD & 69.54 & 86.98 & 98.23 & 109.44 \\
\hline \multirow[t]{2}{*}{ TOTAL } & Mean & 169.76 & 145.16 & 190.89 & 187.47 \\
\hline & SD & 54.15 & 57.89 & 58.18 & 81.29 \\
\hline
\end{tabular}

SD: Standard Desviation. 
ideal conditions and the clinical conditions of the patients were not taken into account.

\section{Conclusion}

Digital impressions with intraoral scanners are an alternative to the conventional techniques in daily practice, obtaining favourable results in the marginal adaptation. More studies are necessary to evaluate the adaptation of the composite overlays.

\section{Conflict of interest}

Authors declare that they have no financial or personal interest that could influence in the results of the study.

\section{References}

1. Ozakar-Ilday N, Zorba YO, Yildiz M, Erdem V, Seven N, et al. (2013) Three-year clinical performance of two indirect composite inlays compared to direct composite restorations. Med Oral Patol Oral Cir Bucal 18: e521-528. [Crossref]

2. Soares CJ, Celiberto L, Dechichi P, Fonseca RB, Martins LR (2005) Marginal integrity and microleakage of direct and indirect composite inlays: SEM and stereomicroscopic evaluation. Braz Oral Res 19: 295-301. [Crossref]

3. Syrek A, Reich G, Ranftl D, Klein C, Cerny B, et al. (2010) Clinical evaluation of allceramic crowns fabricated from intraoral digital impressions based on the principle of active wavefront sampling. $J$ Dent 38: 553-559. [Crossref]

4. Duquia Rde C, Osinaga PW, Demarco FF, de V Habekost L, Conceição EN (2006)
Cervical microleakage in MOD restorations: in vitro comparison of indirect and direct composite. Oper Dent 31: 682-687. [Crossref]

5. Zarrati S1, Mahboub F (2010) Marginal adaptation of indirect composite, glass-ceramic inlays and direct composite: an in vitro evaluation. J Dent (Tehran) 7: 77-83. [Crossref]

6. van der Meer WJ, Andriessen FS, Wismeijer D, Ren Y (2012) Application of intraoral dental scanners in the digital workflow of implantology. PLoS One 7: e43312. [Crossref]

7. Patzelt SB, Lamprinos C2, Stampf S3, Att W4 (2014) The time efficiency of intraoral scanners: an in vitro comparative study. J Am Dent Assoc 145: 542-551. [Crossref]

8. Lee SJ, Gallucci GO (2013) Digital vs. conventional implant impressions: efficiency outcomes. Clin Oral Implants Res 24: 111-115. [Crossref]

9. Almeida e Silva JS, Erdelt K, Edelhoff D, Araújo É, Stimmelmayr M, et al. (2014) Marginal and internal fit of four-unit zirconia fixed dental prostheses based on digital and conventional impression techniques. Clin Oral Investig 18: 515-523. [Crossref]

10. Ting-Shu S, Jian S1 (2015) Intraoral Digital Impression Technique: A Review. $J$ Prosthodont 24: 313-321. [Crossref]

11. Seelbach P, Brueckel C, Wöstmann B (2013) Accuracy of digital and conventional impression techniques and workflow. Clin Oral Investig 17: 1759-1764. [Crossref]

12. Zaruba M, Kasper R, Kazama R, Wegehaupt FJ, Ender A, et al. (2014) Marginal adaptation of ceramic and composite inlays in minimally invasive mod cavities. Clin Oral Investig 18: 579-587. [Crossref]

13. Milleding P (1992) Microleakage of indirect composite inlays. An in vitro comparison with the direct technique. Acta Odontol Scand 50: 295-301. [Crossref]

14. Ng J, Ruse D2, Wyatt C3 (2014) A comparison of the marginal fit of crowns fabricated with digital and conventional methods. J Prosthet Dent 112: 555-560. [Crossref]

Copyright: $\odot 2016$ Llácer VJF. This is an open-access article distributed under the terms of the Creative Commons Attribution License, which permits unrestricted use, distribution, and reproduction in any medium, provided the original author and source are credited. 